\title{
Contribuição genética ótima aplicada à seleção de ovinos Santa Inês
}

\author{
Natanael Pereira da Silva Santos ${ }^{(1)}$, José Lindenberg Rocha Sarmento(2), Roberto Carvalheiro(3), \\ José Elivalto Guimarães Campelo(2), Wandrick Hauss de Sousa(4), Luiz Antônio Silva Figueiredo Filho(5), \\ Aurino de Araújo Rego Neto(6) e Daniel Biagiotti ${ }^{(7)}$
}

\begin{abstract}
(1)Universidade Federal do Piauí, Campus Professora Cinobelina Elvas, BR-135, Km 3, CEP 64900-000 Bom Jesus, PI, Brasil. E-mail: natanaelpss@hotmail.com ${ }^{(2)}$ Universidade Federal do Piauí, Centro de Ciências Agrárias, Departamento de Zootecnia, Campus da Socopo, CEP 64049-550 Teresina, PI, Brasil. E-mail: sarmento@ufpi.edu.br, jelivalto@hotmail.com (3)Universidade Estadual Paulista, Faculdade de Ciências Agrárias e Veterinárias, Via de Acesso Professor Paulo Donato Castellane, s/no, CEP 14884-900 Jaboticabal, SP, Brasil. E-mail: rcar@fcav.unesp.br ${ }^{(4)}$ Empresa Estadual de Pesquisa Agropecuária da Paraíba S/A, Rua Eurípedes Tavares, № 210, Tambiá, CEP 58013-290 João Pessoa, PB, Brasil. E-mail: wandrick@gmail.com ${ }^{(5)}$ Instituto Federal do Maranhão, Campus Timon, Avenida Luis Firmino de Sousa, no 3.907, Multirão, CEP 65635-468 Timon, MA, Brasil. E-mail: luiz_medvet@hotmail.com ${ }^{(6)}$ Universidade Estadual do Piauí, Avenida Dom Pedro II, no 629, Campus XI Corrente, CEP 64980-000 Corrente, PI, Brasil. E-mail: aurinorego@hotmail.com ${ }^{(7)}$ Colégio Agrícola de Bom Jesus, Campus Professora Cinobelina Elvas, BR-135, Km 3, CEP 64900-000 Bom Jesus, PI, Brasil. Email: danielbiagiotti@hotmail.com
\end{abstract}

Resumo - O objetivo deste trabalho foi avaliar estratégias de seleção simulada pela contribuição genética ótima quanto ao ganho genético, com restrição sobre a coancestria, para a característica peso aos 84 dias idade, em ovinos Santa Inês. As análises foram definidas conforme um algoritmo de evolução diferencial, que otimizou uma função-objetivo composta pelo mérito genético e a coancestria média dos animais candidatos à reprodução. A estratégia definida como ótima indicou a utilização de 19 carneiros, o que resultaria em ganho médio esperado de 1,1259 unidades de desvio-padrão, e coancestria igual a 0,0249 (estratégia 5). Em comparação à seleção com base apenas no valor genético, a estratégia ótima reduziu a coancestria em $12 \%$, e o ganho genético, em apenas $3 \%$. A seleção de ovinos pela contribuição genética ótima oferece diferentes níveis de ganho genético, que são atingíveis a partir de restrições sobre a coancestria. Assim, é possível minimizar a coancestria, ou restringi-la em um valor pré-definido, e maximizar o ganho genético simultaneamente com o uso da contribuição genética ótima.

Termos para indexação: coancestria, endogamia, evolução diferencial, ganho genético, resposta à seleção.

\section{Optimum genetic contribution applied to the selection of Santa Ines sheep}

\begin{abstract}
The objective of this work was to evaluate strategies of simulated selection by optimum contribution for the genetic gain, with restriction on coancestry, for the weight trait of 84-day old Santa Ines sheep. The analyses were performed using a differential evolution algorithm, which optimized an objective function accounting for average coancestry of the candidate animals for reproduction and genetic merit. The optimal selection strategy indicated the use of 19 rams, which would result in an expected average gain of 1.1259 units of standard deviation, and coancestry equal to 0.0249 (strategy 5). Compared to the selection based only on breeding values, the optimum strategy reduced the coancestry by $12 \%$, and the genetic gain by only $3 \%$. The selection of sheep by the optimum contribution offers different levels of genetic gain, which are achievable from restrictions on the coancestry. Therefore, it is possible to minimize the coancestry, or restrict it to a predefined value, and maximize the genetic gain while using the optimum contribution.
\end{abstract}

Index terms: coancestry, inbreeding, differential evolution, genetic gain, response to selection.

\section{Introdução}

A resposta à seleção em programas de melhoramento genético é substancialmente aumentada em consequência da melhoria contínua dos métodos de avaliação genética e da alta intensidade de seleção. Porém, o excesso de uso de animais selecionados pode acarretar a perda da variabilidade genética do rebanho (Sánchez et al., 2003).
Acasalamentos e cruzamentos com planejamento inadequado têm ocasionado perda de variabilidade genética dos animais da raça Santa Inês localmente adaptados. Isso reforça e torna essencial o desenvolvimento de ferramentas e estratégias para a manutenção da variabilidade inter-racial, a qual evita a extinção das raças, e intrarracial, que evita a erosão genética (Menezes et al., 2006). 
O parentesco entre os indivíduos está diretamente relacionado à diversidade genética da população e à endogamia esperada na próxima geração, pois os animais têm genes iguais, mas podem diferir em seus alelos. O controle da endogamia e a consequente manutenção da variabilidade genética em longo prazo não são garantidas, quando restrições são feitas apenas sobre a endogamia da futura progênie (Breda et al., 2004). Neste caso, a estratégia mais apropriada seria considerar restrições à coancestria, a fim de aumentar a resposta à seleção em longo prazo (Kinghorn, 2011; $\mathrm{Oh}, 2012)$.

A aplicação do método da contribuição genética ótima se apresenta como uma importante opção para definir a intensidade de uso dos animais candidatos à reprodução, conforme um índice (função-objetivo) que combina o mérito genético e a coancestria dos candidatos à seleção (Eisen, 2007). Assim, a solução ideal é expressa em contribuições genéticas dos candidatos selecionados para a próxima geração, que é proporcional ao número ideal de descendentes para cada candidato.

Em termos de praticidade, a maioria dos programas de auxílio à seleção são ineficientes pela difícil manipulação, além de demandarem alta capacidade computacional, o que pode criar uma barreira para sua utilização. Uma alternativa promissora de busca otimizada, para a definição da contribuição genética ótima, é a adoção de um algoritmo com base na evolução diferencial (differential evolution - DE) (Storn \& Price, 1997), que tem fundamentação nos mecanismos da evolução natural das espécies. Favorável ao algorítimo DE está o fato de este possibilitar um exame simultâneo da solução, em diferentes regiões no espaço de busca, fazendo-se uso de uma "população de soluções candidatas" para o equacionamento do problema.

O objetivo deste trabalho foi avaliar estratégias de seleção simulada pela contribuição genética ótima quanto ao ganho genético, com restrição sobre a coancestria, para a característica peso aos 84 dias idade, em ovinos Santa Inês.

\section{Material e Métodos}

Os dados utilizados neste estudo provêm de dois rebanhos experimentais de ovinos da raça Santa Inês, pertencentes à Empresa Estadual de Pesquisa
Agropecuária da Paraíba (Emepa-PB), relativos ao período de 1992 a 2014.

$\mathrm{O}$ arquivo inicial consistia de 3.365 registros com informações ponderais de animais, mensuradas a cada 28 dias, em média, do nascimento aos 196 dias de idade. Consideraram-se para análise as seguintes informações sobre os cordeiros pesados aos 84 dias de idade (P84): nascidos de parto simples ou múltiplo; com peso médio dentro da média de todos os animais de mesmo grupo de contemporâneos $(\mathrm{GC}) \pm$ três e meio desvios-padrão; e que pertenciam a GCs com no mínimo quatro animais. A definição dos GCs incluiu: rebanho, ano e período de nascimento, sexo e tipo de nascimento do cordeiro. Definiram-se dois períodos de nascimento: o chuvoso, de fevereiro a julho; e o seco, de agosto a janeiro. Permaneceram no arquivo animais com pai e mãe conhecidos, tendo restado 2.970 animais diferentes na matriz de parentesco para as análises.

Consideraram-se aptos à reprodução os animais machos vivos, com idade entre 1 e 3 anos e com valores genéticos preditos (EBVs) maiores que 0,2. Assim, restou para a análise da contribuição genética ótima o total de 122 machos. Impôs-se a restrição de que cada macho pudesse acasalar no máximo 20 vezes, já que o manejo reprodutivo praticado nas fazendas experimentais é de monta natural controlada. Além disso, fixou-se o número máximo de possíveis acasalamentos em 290, número que corresponde ao total de fêmeas aptas à reprodução disponíveis no rebanho (fêmeas vivas com mais de 12 meses de vida, não prenhes e que pariram há mais de 3 meses).

As análises da contribuição genética ótima de cada um dos 122 carneiros candidados à seleção foi definida conforme a solução fornecida pelo programa DEforOC (Differential evolution for optimum contribution) (Carvalheiro et al., 2010), escrito em linguagem Fortran, que consistiu em gerar aleatoriamente uma população de soluções possíveis, composta pela intensidade de uso de cada carneiro candidato.

Os parâmetros da evolução diferencial utilizados foram: tamanho da população, 244 ( 2 x número de candidatos); taxa de recombinação, $\mathrm{CR}=0,5$; fator de mutação, $\mathrm{F}=0,1$ (ou $\mathrm{F}=1,0$ a cada 4 gerações). A convergência do processo evolutivo foi assumida quando a amplitude e o desvio absoluto médio dos valores adaptativos dos indivíduos de uma geração foram menores do que $1 \times 10^{-6}$, ou a melhor solução da geração $5 \times 10^{4}$. 
A função-objetivo (OF), que definiu os valores adaptativos dos indivíduos, foi composta pelo mérito genético e pela coancestria média dos animais candidatos à reprodução e pode ser descrita como $\mathrm{OF}=\lambda_{1} \mathrm{c}^{\prime} \mathrm{EBV}+\lambda_{2} \mathrm{c}^{\prime} \mathrm{Ac}$, em que : c'EBV é a média do mérito genético predito dos pais selecionados (reflete o ganho genético); c'Ac é a coancestria média dos animais em reprodução (reflete a endogamia em longo prazo); e $\lambda_{1}$ e $\lambda_{2}$ são os ponderadores associados aos respectivos componentes. O vetor c' representa a contribuição genética ótima dos candidatos à reprodução (o sinal 'indica vetor transposto); EBV é o vetor da melhor predição linear não viesada (Blup best linear unbiased prediction), que corresponde aos valores genéticos; e A é a matriz de numeradores do coeficiente de parentesco de Wright.

Consideraram-se diferentes ponderadores sobre a coancestria, e avaliou-se a consequência da aplicação do algoritmo desenvolvido na seleção dos carneiros. Para tal, avaliaram-se 13 estratégias de seleção com base em restrições impostas aos ponderadores $\left(\lambda_{1} \mathrm{e} \lambda_{2}\right)$. A estratégia 1 contemplou somente o mérito genético predito dos pais selecionados $\left(\lambda_{1}=1\right.$ e $\left.\lambda_{2}=0\right)$, que apontaria o maior ganho genético, se a seleção fosse realizada apenas considerando-se o EBV dos animais. Nas estratégias 2 a 12, fixou-se o valor do ponderador do mérito genético predito dos pais selecionados em $1\left(\lambda_{1}=1\right)$, e restringiram-se os valores da coancestria com números crescentes e negativos $\left(\lambda_{2}\right.$ variando de -5 a -800), com a finalidade de se observar o nível de restrição da endogamia que proporcione menores perdas quanto ao ganho genético. A estratégia 13 foi utilizada para identificar a redução máxima possível da coancestria na população em estudo $\left(\lambda_{1}=0\right.$ e $\left.\lambda_{2}=-1\right)$. $\mathrm{O}$ resultado deste procedimento de otimização é o vetor c' das contribuições para a próxima geração de candidatos a qualquer geração particular (intensidade de uso do animal).

\section{Resultados e Discussão}

O nível de endogamia do rebanho é de 0,066 (6\%), com 236 animais endogâmicos que correspondem a $8 \%$ do total de animais no pedigree (2.790 animais). Assim, ao se admitir que o índice de endogamia superior a $10 \%$ representa risco elevado para a ocorrência de depressão por endogamia (Paiva et al., 2011), o rebanho merece atenção a este indicador. $O$ índice de endogamia observado pode ser resultante do uso marcante de poucos reprodutores, o que pode promover o aumento de laços genéticos entre eles.

Encontraram-se soluções com os valores de ponderação adotados (estratégias 1 a 13) na população (Tabela 1). As funções-objetivo otimizadas nas estratégias de 2 a 13 restringem a coancestria dos animais em reprodução com a finalidade de controlar a endogamia em longo prazo. Níveis aceitáveis de endogamia são difíceis de serem determinados e foram discutidos por Bijma et al. (2001), que indicaram que a depressão por endogamia é provavelmente a característica mais importante ligada ao melhoramento genético e que, apesar do conhecimento detalhado dos parâmetros relevantes para se determinar o nível da restrição, esta não deve exceder $1 \%$ por geração.

A análise que contemplou apenas o mérito genético predito dos pais selecionados (estratégia 1) foi incluída, para validar o programa e servir de parâmetro de comparação - contribuição de 20 descendentes, de cada um dos 14 melhores carneiros, e 10 descendentes para o subsequente, no total de 290 acasalamentos. A otimização da função-objetivo que contemplou apenas o mérito genético resultou em um valor genético médio esperado de 1,1620 da futura progênie, que corresponde ao valor máximo de ganho genético que poderia ser obtido em curto prazo, com a seleção dos animais com base no maior EBV (Tabela 1). Entretanto, a seleção dos animais exclusivamente pelo EBV (estrategia 1) maximiza a resposta à seleção apenas em relação à geração seguinte, já que estes valores são as soluções Blup.

A estratégia 1, em comparação às demais estratégias, resultou no maior nível de coancestria $(0,0283)$ e no uso de 15 carneiros (maior intensidade de seleção). Esse resultado pode ser explicado pelo fato de que o Blup usa informações de todos os parentes para predizer os EBVs dos animais. Assim, quando se utilizam informações de toda a família, aquelas que apresentam indivíduos com melhor desempenho têm mais chance de serem selecionadas em conjunto $\mathrm{e}$, consequentemente, os futuros acasalamentos desses animais podem elevar a taxa de endogamia da população. Deste modo, a consequência direta da elevada coancestria dos animais em reprodução é o aumento da taxa de endogamia que, por sua vez, pode ocasionar prejuízo à variabilidade e ao progresso genético em médio e longo prazo (Weigel, 2001).

A estratégia definida como ótima, ou seja, aquela que permite minimizar os efeitos da coancestria, mantendo 
um nível de ganho genético aceitável para o rebanho, indica a seleção de 19 carneiros, o que resultaria em progênies com índice médio esperado de 1,1259 unidades de desvio-padrão e 0,0249 de coancestria (estratégia 5).

Nota-se que as soluções fornecidas pela evolução diferencial para as estratégias 3, 4 e 5 indicam o uso de 19 carneiros, assim como as estratégias 10, $11 \mathrm{e}$ 12 indicam o uso de 31 animais como reprodutores. Deve-se ressaltar que, apesar da indicação da mesma quantidade de animais a serem selecionados nestes dois grupos de estratégias, os animais não são os mesmos, assim como a intensidade de uso de cada animal não é necessariamente igual para cada estratégia. Isso deixa claro que a substituição de um reprodutor candidato por outro e a quantidade de descendentes que o mesmo animal deixará no rebanho podem implicar na diminuição de ganho genético, o que também se pode observar quanto à coancestria média no rebanho, com as mesmas estratégias mencionadas.

A tentativa de controle da endogamia, pela imposição de restrições das estratégias 8 a 12, quando comparadas entre si, além de apresentar redução do ganho genético esperado, praticamente não reduziu a coancestria média dos animais, se usados na reprodução. Porém, observase sensível diferença, quanto ao maior ganho genético, na hipótese de a seleção ser realizada adotando-se a estratégia 8.
Na seleção pelas estratégias 2 a 13, observa-se aumento do número de carneiros selecionados e a redução do mérito genético esperado dos candidatos selecionados, quando comparada à seleção pela estratégia 1 . Ou seja, o aumento do número de carneiros selecionados reflete o efeito da minimização da coancestria. Com isso, a redução da coancestria reduziria a futura endogamia e, em longo prazo, aumentaria o ganho genético (Sonesson \& Meuwisson, 2001).

Observa-se que a estruturação dos rebanhos com relacionamentos menos extremos pode melhorar a seleção dos candidatos a reprodutores na próxima geração, pela contribuição genética ótima. Este resultado deixa clara a diferença entre as seleções por truncamento e por contribuição genética ótima, no que tange ao uso e à quantidade de reprodutores selecionados. Considerando-se que, na seleção por truncamento, são atribuídas as mesmas proporções de acasalamentos a todos os indivíduos com valor genético acima de um certo ponto de corte (Daetwyler et al., 2007), com a contribuição genética ótima é possível variar o uso entre os candidatos selecionados (Kinghorn, 2011).

A estratégia 5 apresenta-se como excelente alternativa de seleção para o rebanho, pois implicou em redução da coancestria em $12 \%$ e redução de ganho genético de apenas $3 \%$ em relação à estratégia 1 (Tabela 2).

Tabela 1. Estratégias de seleção pela contribuição genética ótima, com uso da evolução diferencial, em ovinos da raça Santa Inês.

\begin{tabular}{|c|c|c|c|c|c|c|c|c|}
\hline Estratégia & $\lambda_{1}$ & $\lambda_{2}$ & N_ger & CPU_time & max_OF & c'EBV & $\mathrm{c}^{\prime} \mathrm{Ac}$ & N_sire \\
\hline 1 & 1 & 0 & 3337 & 2,04 & 1,1620 & 1,1620 & 0,0283 & 15 \\
\hline 2 & 1 & -5 & 4585 & 4,11 & 0,8844 & 1,1511 & 0,0267 & 17 \\
\hline 3 & 1 & -7 & 5588 & 6,56 & 0,7792 & 1,1406 & 0,0258 & 19 \\
\hline 4 & 1 & -8 & 5200 & 5,04 & 0,7279 & 1,1350 & 0,0254 & 19 \\
\hline 5 & 1 & -10 & 3833 & 2,78 & 0,6274 & 1,1259 & 0,0249 & 19 \\
\hline 6 & 1 & -20 & 4956 & 4,48 & 0,1448 & 1,0878 & 0,0236 & 24 \\
\hline 7 & 1 & -30 & 2781 & 1,12 & $-0,3239$ & 1,0672 & 0,0232 & 25 \\
\hline 8 & 1 & -50 & 5629 & 6,00 & $-1,2407$ & 1,0295 & 0,0227 & 27 \\
\hline 9 & 1 & -80 & 2858 & 1,45 & $-2,5945$ & 0,9993 & 0,0226 & 29 \\
\hline 10 & 1 & -200 & 2997 & 1,53 & $-7,9596$ & 0,9662 & 0,0225 & 31 \\
\hline 11 & 1 & -500 & 14769 & 22,85 & $-21,3385$ & 0,9519 & 0,0224 & 31 \\
\hline 12 & 1 & -800 & 3256 & 1,98 & $-34,711$ & 0,9477 & 0,0223 & 31 \\
\hline 13 & 0 & -1 & 2554 & 1,07 & $-0,0446$ & 0,9468 & 0,0223 & 33 \\
\hline
\end{tabular}

$\lambda_{1}$, ponderador para o mérito genético; $\lambda_{2}$, ponderador para coancestria; N_ger, número de gerações para a convergência do algoritmo; CPU_time, tempo de processamento da análise em segundos; max_OF, função-objetivo maximizada; c’EBV, média do mérito genético predito dos pais selecionados (reflete o ganho genético); c'Ac, coancestria média dos animais em reprodução (reflete a endogamia em longo prazo); N_sire, número de carneiros selecionados para acasalamento. 
Alguns trabalhos destacam o efeito depressor da endogamia sobre características de peso em ovinos, como o realizado por Pedrosa et al. (2010). Norberg \& Sørensen (2007), observaram redução de peso de 0,$011 ; 0,0082$ e $0,0088 \mathrm{~kg}$ a cada $1 \%$ de endogamia, em ovinos das raças Texel, Shropshire e Oxford Down, respectivamente. Selvaggi et al. (2010), relataram perda de $0,019 \mathrm{~kg}$ de peso ao nascimento e de $0,031 \mathrm{~kg}$ de peso à desmama, a cada $1 \%$ de aumento da endogamia, em estudos sobre a raça Leccese. Porém, Hossein-Zadeh (2012) não encontrou diferenças significativas de desempenho de ovelhas da raça Moghani até os $10 \%$ de endogamia, quanto aos pesos ao nascimento e aos 3 meses de idade.

Quanto a outros aspectos favoráveis à metodologia pesquisada, argumenta-se que, na otimização por evolução diferencial, a convergência foi rápida (em média $4 \mathrm{~s}$ ), o que pode indicar maior eficiência de uma série de algoritmos evolucionários complexos (Mayer et al., 2005). O programa DEforOC pode ser considerado uma ferramenta de gestão para o planejamento de programas de melhoramento genético do nível populacional de ovinos, para maximizar o ganho genético, enquanto controla o aumento de taxas futuras de endogamia.

Tabela 2. Diferenças, quanto ao ganho genético e à endogamia em longo prazo, considerando-se estratégias de seleção pela contribuição genética ótima, em ovinos Santa Inês.

\begin{tabular}{lccccccc}
\hline Estratégia & \multicolumn{3}{c}{$\mathrm{c}^{\prime} E B V$} & & \multicolumn{3}{c}{$\mathrm{c}$ 'Ac } \\
\cline { 2 - 3 } \cline { 7 - 8 } & $\mathrm{d}$ & $\mathrm{d}(\%)$ & $\mathrm{a}(\%)$ & & $\mathrm{d}$ & $\mathrm{d}(\%)$ & $\mathrm{a} \mathrm{( \% )}$ \\
\hline 1 & - & - & - & - & - & - \\
2 & 0,0109 & 0,94 & 0,94 & 0,0016 & 5,65 & 5,65 \\
3 & 0,0105 & 0,90 & 1,84 & & 0,0009 & 3,18 & 8,83 \\
4 & 0,0056 & 0,48 & 2,32 & & 0,0004 & 1,41 & 10,25 \\
5 & 0,0091 & 0,78 & 3,11 & & 0,0005 & 1,77 & 12,01 \\
6 & 0,0381 & 3,28 & 6,39 & & 0,0013 & 4,59 & 16,61 \\
7 & 0,0206 & 1,77 & 8,16 & & 0,0004 & 1,41 & 18,02 \\
8 & 0,0377 & 3,24 & 11,40 & & 0,0005 & 1,77 & 19,79 \\
9 & 0,0302 & 2,60 & 14,00 & & 0,0001 & 0,35 & 20,14 \\
10 & 0,0331 & 2,85 & 16,85 & & 0,0001 & 0,35 & 20,49 \\
11 & 0,0143 & 1,23 & 18,08 & & 0,0001 & 0,35 & 20,85 \\
12 & 0,0042 & 0,36 & 18,44 & & 0,0001 & 0,35 & 21,20 \\
13 & 0,0009 & 0,08 & 18,52 & 0,0000 & 0,00 & 21,20 \\
\hline
\end{tabular}

c'EBV, média do mérito genético predito dos pais selecionados (reflete o ganho genético); c'Ac, coancestria média dos animais em reprodução (reflete a endogamia em longo prazo); d, diferença em relação à estratégia anterior; a, acumulado.
Vale ressaltar que o método testado também pode ser aplicado para avaliar e controlar a taxa de endogamia em programas de conservação de animais em risco de extinção, em que o mérito genético não é o fator mais importante. Apesar de a endogamia ser inevitável em programas fechados de seleção, seu aumento precisa ser restrito, para reduzir seus efeitos negativos.

Os resultados denotam a exigência de informação confiável do pedigree, deixando-se evidente que é necessária a correta coleta dos dados. Assim, por mais restrita que seja a quantidade de informações, estas devem ser bem estruturadas, a fim de se evitarem erros na seleção dos animais. Assim, o método da contribuição genética ótima pode ser usado para gerar uma medida da eficiência de um esquema de seleção, como afirmado por Fimland (2007).

Destaca-se, ainda, que nenhuma tentativa foi realizada com a finalidade de se obterem os fatores de ponderação ideais, mas, apenas observar o impacto da estratégia que pode apresentar a melhor resposta para o sistema de criação, na geração seguinte. Assim, a definição do ponto ótimo foi realizada em razão dos objetivos da seleção, ou seja, reduzir os níveis de coancestria no rebanho, com perdas mínimas de ganho genético.

\section{Conclusões}

1. A seleção de ovinos Santa Inês pela contribuição genética ótima oferece diferentes níveis de ganho genético, que são atingíveis a partir de restrições sobre a coancestria.

2. É possível minimizar a coancestria, ou restringi-la em um valor pré-definido, e maximizar o ganho genético simultaneamente com o uso da contribuição genética ótima.

\section{Agradecimentos}

À Empresa Estadual de Pesquisa Agropecuária da Paraíba (Emepa), pela concessão de uso dos dados.

\section{Referências}

BIJMA, P.; VAN ARENDONK, J.A.M.; WOOLLIAMS, J.A. Predicting rates of inbreeding for livestock improvement schemes. Journal of Animal Science, v.79, p.840-853, 2001.

BREDA, F.C.; EUCLYDES, R.F.; PEREIRA, C.S.; TORRES, R. de A.; CARNEIRO, P.L.S.; SARMENTO, J.L.R.; TORRES 
FILHO, R. de A.; MOITA, A.K.F. Endogamia e limite de seleção em populações selecionadas obtidas por simulação. Revista Brasileira de Zootecnia, v.33, p.2017-2025, 2004. Supl. 2. DOI: 10.1590/S1516-35982004000800013.

CARVALHEIRO, R.; QUEIROZ, S.A. de; KINGHORN, B. Optimum contribution selection using differential evolution. Revista Brasileira de Zootecnia, v.39, p.1429-1436, 2010. DOI: 10.1590/S1516-35982010000700005.

DAETWYLER, H.D.; VILLANUEVA, B.; BIJMA, P.; WOOLLIAMS, J.A. Inbreeding in genome-wide selection. Journal of Animal Breeding and Genetics, v.124, p.369-376, 2007. DOI: 10.1111/j.1439-0388.2007.00693.x.

EISEN, E.J. Animal breeding: What does the future hold? Asian-Australasian Journal of Animal Science, v.20, p.453-460, 2007. DOI: 10.5713/ajas.2007.453.

FIMLAND, E. Genetic diversity and sustainable management of animal genetic resources, globally. Animal Genetic Resources Information, v.41, p.45-52, 2007.

HOSSEIN-ZADEH, N.G. Inbreeding effects on body weight traits of Iranian Moghani sheep. Archiv Tierzucht, v.55, p.171-178, 2012.

KINGHORN, B.P. An algorithm for efficient constrained mate selection. Genetics Selection Evolution, v.43, p.4-12, 2011. DOI: 10.1186/1297-9686-43-4.

MAYER, D.G.; KINGHORN, B.P.; ARCHER, A.A. Differential Evolution - an easy and efficient evolutionary algorithm for model optimization. Agricultural Systems, v.83, p.315-328, 2005. DOI: 10.1016/j.agsy.2004.05.002.

MENEZES, M.P.C.; MARTINEZ, A.M.; RIBEIRO, M.N.; PIMENTA FILHO, E.C.; BERMEJO, J.V.D. Caracterização genética de raças caprinas nativas brasileiras utilizando-se 27 marcadores microssatélites. Revista Brasileira de Zootecnia, v.35, p.1336-1341, 2006. DOI: 10.1590/S1516-35982006000500012.

NORBERG, E.; SØRENSEN, A.C. Inbreeding trend and inbreeding depression in the Danish populations of Texel, Shropshire, and
Oxford Down. Journal of Animal Science, v.85, p.299-304, 2007. DOI: $10.2527 /$ jas.2006-257.

$\mathrm{OH}$, S.-H. Evaluation of optimum genetic contribution theory to control inbreeding while maximizing genetic response. Asian-Australasian Journal of Animal Science, v.25, p.299-303, 2012.

PAIVA, S.R.; FACÓ, O.; FARIA, D.A.; LACERDA, T.; BARRETTO, G.B.; CARNEIRO, P.L.S.; LOBO, R.N.B.; MCMANUS, C. Molecular and pedigree analysis applied to conservation of animal genetic resources: the case of Brazilian Somali hair sheep. Tropical Animal Health and Production, v.43, p.1449-1457, 2011. DOI: 10.1007/s11250-011-9873-6.

PEDROSA, V.B.; SANTANA JR., M.L.; OLIVEIRA, P.S.; ELER, J.P.; FERRAZ, J.B.S. Population structure and inbreeding effects on growth traits of Santa Inês sheep in Brazil. Small Ruminant Research, v.93, p.135-139, 2010. DOI: 10.1016/j. smallrumres.2010.05.012

SÁNCHEZ, L.; BIJMA, P.; WOOLLIAMS, J.A. Minimizing inbreeding by managing genetic contributions across generations. Genetics, v.164, p.1589-1595, 2003.

SELVAGGI, M.; DARIO, C.; PERETTI, V.; CIOTOLA, F.; CARNICELLA, D.; DARIO, M. Inbreeding depression in Laccese sheep. Small Ruminant Research, v.89, p.42-46, 2010. DOI: 10.1016/j.smallrumres.2009.12.005.

SONESSON, A.K.; MEUWISSEN, T.H.E. Minimization of rate of inbreeding for small populations with overlapping generation. Genetics Research, v.77, p.285-292, 2001. DOI: 10.1017/ S0016672301005079.

STORN, R.; PRICE, K. Differential Evolution: a simple and efficient heuristic for global optimization over continuous spaces. Journal of Global Optimization, v.11, p.341-359, 1997. DOI: 10.1023/A:1008202821328.

WEIGEL, K.A. Controlling inbreeding in modern breeding programs. Journal of Dairy Science, v.84, p.E177-E184, 2001. Supplement. DOI: 10.3168/jds.S0022-0302(01)70213-5.

Recebido em 15 de outubro de 2015 e aprovado em 21 de março de 2016 